\title{
Parallel, High-quality Proteomic and Targeted Metabolomic Quantification Using Laser Capture Micro-Dissected Tissues
}

\author{
Shichen Shen ${ }^{1,2}$, Jun Li ${ }^{1,2}$, Shihan Huo ${ }^{1}$, Min $\mathrm{Ma}^{3}$, Xiaoyu Zhu ${ }^{1}$, Sailee Rasam ${ }^{4}$, *, Xiaotao \\ Duan1, *Miao Qu', *Mark A. Titus ${ }^{3,5}$, *Jun Qu ${ }^{1,2}$
}

${ }^{1}$ Department of Pharmaceutical Sciences, SUNY-Buffalo, Buffalo, NY 14214; ${ }^{2}$ New York State Center of Excellence in Bioinformatics \& Life Sciences, Buffalo, NY 14203; ${ }^{2}$ Roswell Park Comprehensive Cancer Institute, Buffalo, NY 14203; ${ }^{4}$ Department of Biochemistry, SUNY-Buffalo, Buffalo, NY 14203; 5 Department of Genitourinary Medical Oncology, University of Texas MD Anderson Cancer Center, Houston, TX 77030; ${ }^{6}$ Department of Neurology, Xuanwu Hospital, Beijing, China 100053

* Corresponding authors

Xiaotao Duan, Ph.D.

E-mail: $\underline{x d u a n @ \text { ncba.ac.cn }}$

Miao Qu, M.D., Ph.D.

E-mail: qumiaotcm@126.com

Mark A. Titus, Ph.D.

Email: MTitus1@mdanderson.org

Jun Qu, Ph.D.

E-mail: junqu@buffalo.edu

\#Current address: State Key Laboratory of Toxicology and Medical Countermeasures, Beijing Institute of Pharmacology and Toxicology, Beijing, China 100850 


\section{TABLE OF CONTENTS}

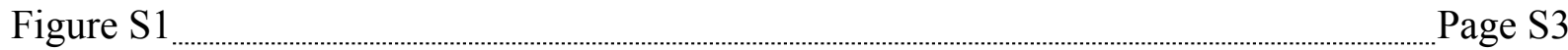

Figure S2

Page S4

Figure S3

Page S5

Figure S4

Page S6

Figure S5

Page S7

Table S1

Page S8

Table S2

Page S9

Table S3\&4

Page S10

Experimental

Page S11

References

Page S18 


\section{SUPPLEMENTARY FIGURES}
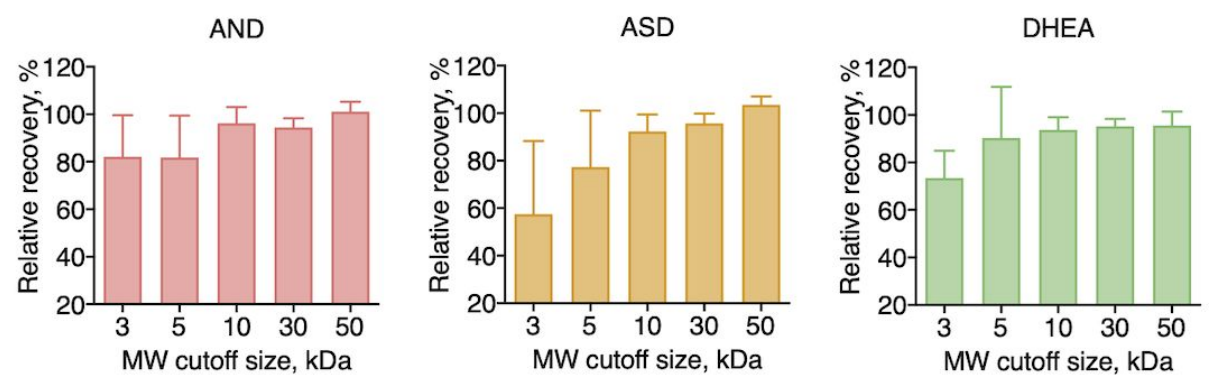

T
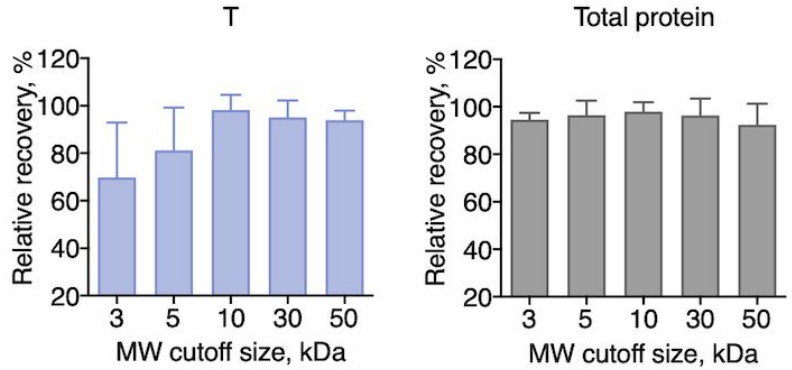

Figure S1. Effects of MW cutoffs on the recovery of androgens and proteins. 


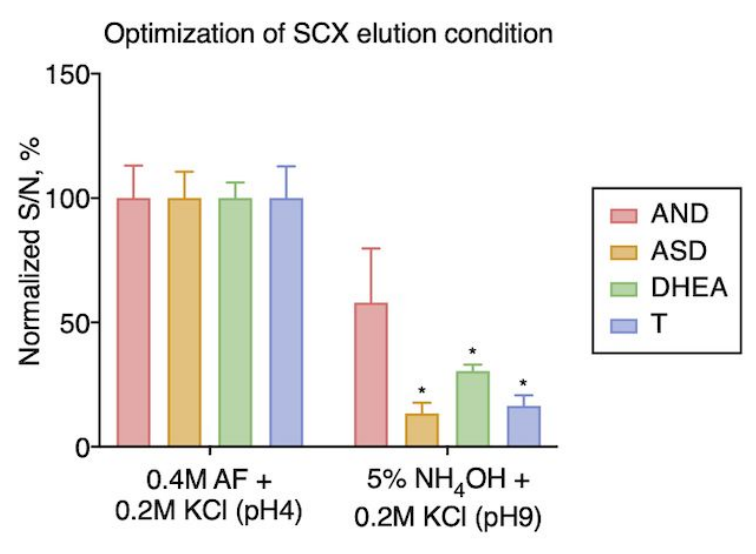

Figure S2. Representative data for optimization of the micro-SCX enrichment of the four GP-androgens. It was found that low-pH elution achieved significantly better signal-to-noise ratio. 
a

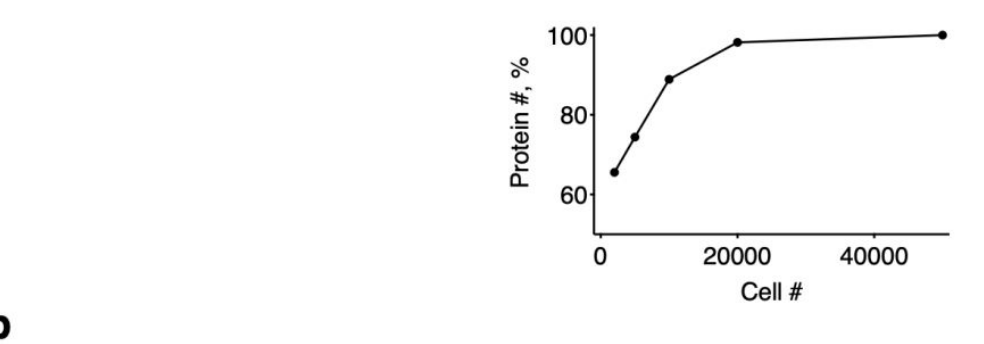

b

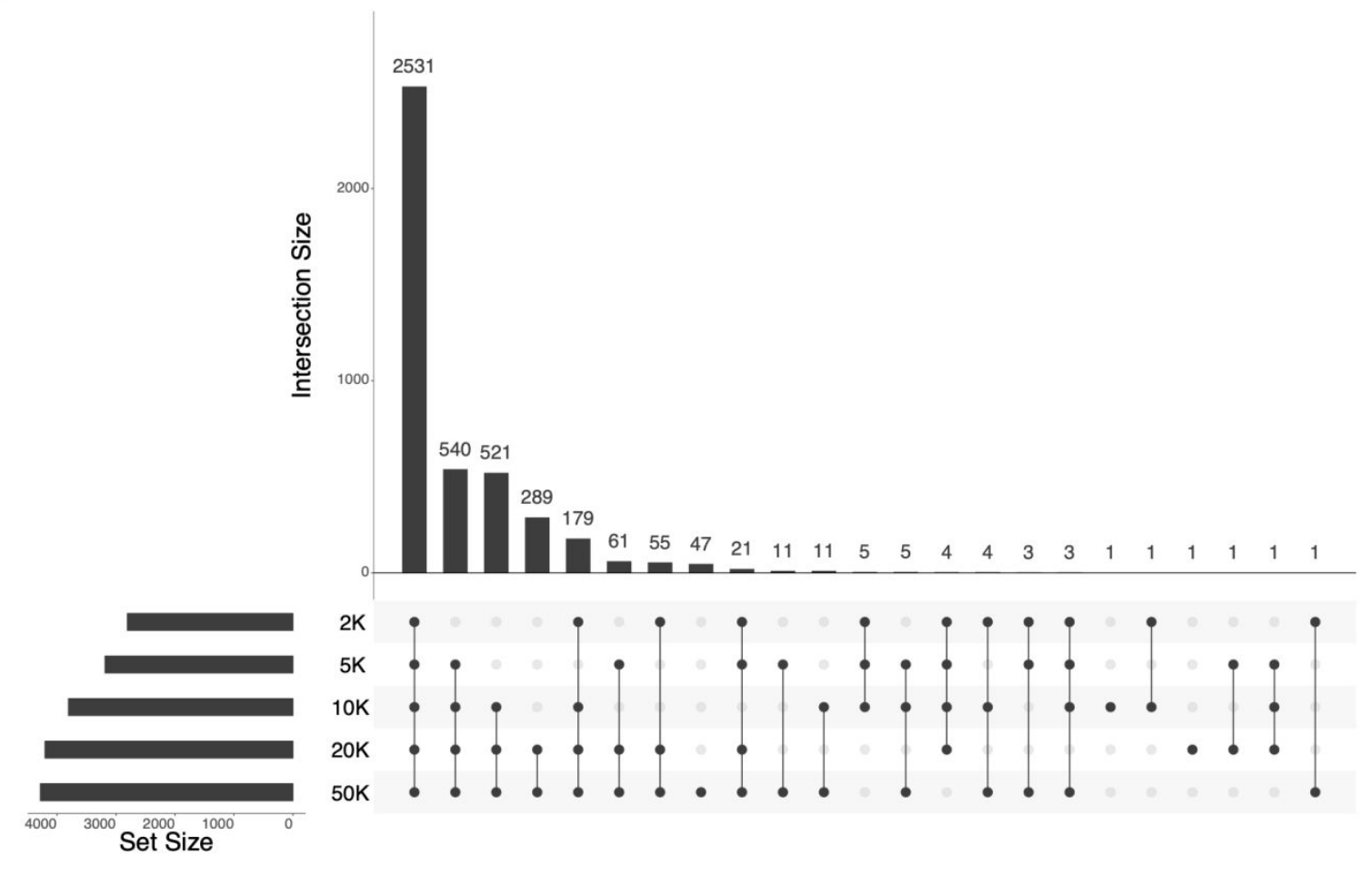

Figure S3. Effects of number of cells per sample on the number of quantified proteins (i.e. $1 \%$ protein FDR, $\geq 2$ peptides identified per protein, $\mathbf{S} / \mathbf{N}>\mathbf{1 0}$ ). a) The number of quantified proteins under different cell numbers; b) UpSetR plot showing the overlap of quantified proteins under different cell numbers (plotted using the UpSetR Shiny App, https://gehlenborglab.shinyapps.io/upsetr/) ${ }^{1}$. 

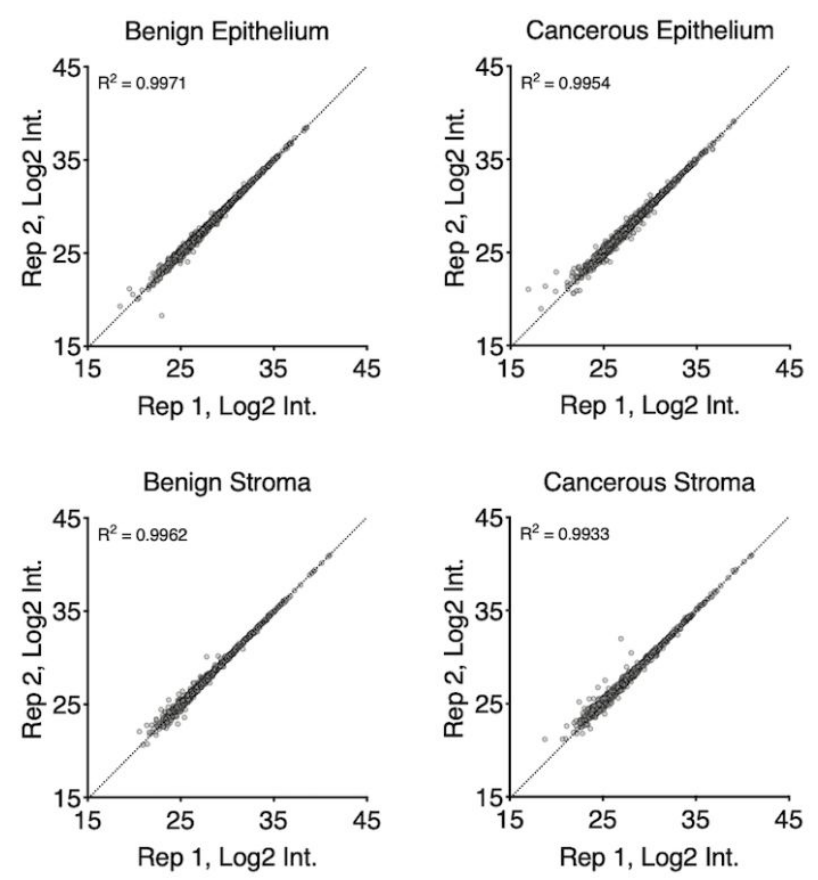

Figure S4. Pearson correlation of protein intensities between two LC-MS replicates of LCM cells. Excellent reproducibility was shown in all 4 regions involved in the proof-of-concept study. 


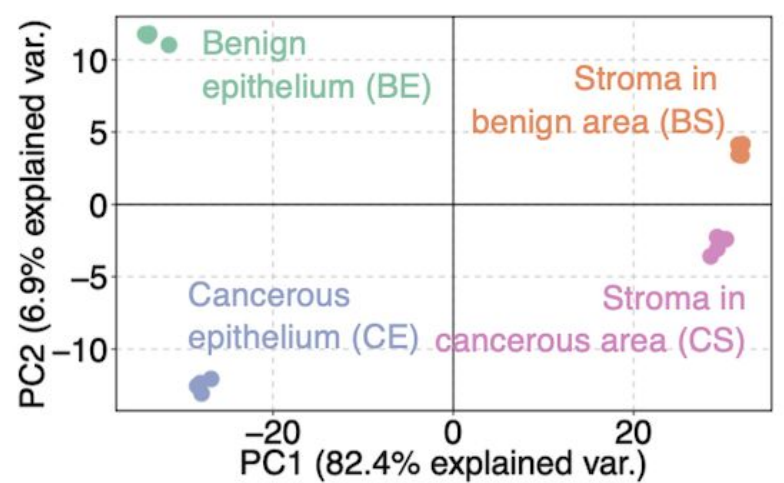

Figure S5. Principal Component Analysis (PCA) of quantified proteins in the PCa patientpaired region: benign epithelium(BE), cancerous epithelium(CE), stroma in benign region(BS), and stroma in cancerous region(CS). The four groups were clearly segregated, suggesting region- and cancer-specific patterns. 


\section{SUPPLEMENTARY TABLES}

Table S1. SRM transitions of the 4 androgens quantified.

\begin{tabular}{lccc}
\hline Analyte & \multicolumn{1}{c}{ Optimized SRM conditions } \\
\hline Androsterone & Precursor Ion $(\mathbf{m} / \mathbf{z})$ & Product ion $(\mathbf{m} / \mathbf{z})$ & $\mathbf{C E}(\mathbf{e V})$ \\
Androsterone-16,16- $d_{2}$ & 424.3 & 345.3 & 25 \\
Androstenedione & 426.3 & 347.3 & 25 \\
Androstenedione-2,3,4- ${ }^{13} C_{3}$ & 277.2 & 237.7 & 18 \\
Dehydroepiandrosterone & 278.7 & 239.2 & 18 \\
Dehydroepiandrosterone-2,2,3,4,4,6- $d_{6}$ & 422.3 & 343.3 & 30 \\
Testosterone & 428.3 & 349.3 & 30 \\
Testosterone-2,3,4- ${ }^{13} C_{3}$ & 422.3 & 343.3 & 30 \\
\hline
\end{tabular}


Table S2. Validation results of the 4 androgens quantified.

\begin{tabular}{|c|c|c|c|c|c|c|c|}
\hline \multirow{3}{*}{ Metabolites } & \multirow{3}{*}{$\begin{array}{l}\text { LOQ (fg/1000 } \\
\text { cells) }\end{array}$} & \multirow{3}{*}{$\begin{array}{c}\text { On-column LOQ } \\
\text { (fg) }\end{array}$} & \multirow{3}{*}{$\begin{array}{c}\text { Range (fg/1000 } \\
\text { cells) }\end{array}$} & \multirow{3}{*}{$\mathbf{R}^{2}$} & \multicolumn{3}{|c|}{ QC } \\
\hline & & & & & Low & Medium & High \\
\hline & & & & & \multicolumn{3}{|c|}{ Accuracy (\%)/CV (\%) } \\
\hline Androsterone (AND) & 5 & 2 & $5-500$ & 0.991 & $98.6 / 10.3$ & $106.4 / 7.8$ & $98.6 / 3.0$ \\
\hline Androstenedione (ASD) & 5 & 2 & $5-500$ & 0.991 & $104.2 / 14.4$ & $95.6 / 3.6$ & $100.8 / 0.6$ \\
\hline $\begin{array}{l}\text { Dehydroepiandrosterone } \\
\text { (DHEA) }\end{array}$ & 5 & 2 & $5-500$ & 0.987 & $101.6 / 7.4$ & $88.7 / 1.1$ & $99.4 / 5.3$ \\
\hline Testosterone $(\mathrm{T})$ & 5 & 2 & $5-500$ & 0.993 & $100.4 / 0.4$ & $98.1 / 11.2$ & $104.4 / 2.0$ \\
\hline
\end{tabular}


Table S3. Quantitative proteomics results of cancerous and benign epithelial cell populations procured by LCM (Available as a separate Microsoft Excel file).

Table S4. Quantitative proteomics results of cancerous and benign stromal cell populations procured by LCM (Available as a separate Microsoft Excel file). 


\section{SUPPLEMENTARY EXPERIMENTAL}

\section{Reagents and materials}

RPMI-1640, charcoal-stripped fetal bovine serum, glutamine, 0.05\% trypsin and other cell culture supplies were obtained from Thermo Fisher Scientific. 10X phosphate buffered saline (PBS) and formic acid (FA) were obtained from VWR International. Sodium deoxycholate (SDC), IGEPAL CA-630, dithiothreitol (DTT), iodoacetamide (IAM) and proteomics grade trypsin were obtained from Sigma Aldrich. Acetone, acetonitrile ( $\mathrm{ACN})$, methanol, Tris, sodium chloride ( $\mathrm{NaCl})$, sodium dodecyl sulfate (SDS), ammonium formate (AF), potassium chloride $(\mathrm{KCl})$, urea, methyl tert-butyl ether (MTBE), Girard's reagent P (GP) and hydrochloric acid ( $\mathrm{HCl}$ ) were obtained from Fisher Chemical. EDTA-free cOmplete protease inhibitor tablets were obtained from Roche. Reference standards of androsterone (AND), androstenedione (ASD), dehydroepiandrosterone (DHEA) and testosterone (T) were obtained from Sigma Aldrich. Stable-isotope-coded internal standards including androsterone-16,16- $d_{2}$, androstenedione-2,3,4- ${ }^{13} \mathrm{C}_{3}$, dehydroepiandrosterone2,2,3,4,4,6- $d_{6}$ and testosterone-2,3,4- ${ }^{13} \mathrm{C}_{3}$ were obtained from Cambridge Isotope Laboratories.

\section{Cell culture and collection}

Homo sapiens androgen-independent prostate cancer cell line C4-2 was obtained from American Type Culture Collection (ATCC \#CRL-3314). Cells were grown in RPMI-1640 supplemented with $10 \%$ charcoal-stripped fetal bovine serum (androgen free) and $1 \%$ glutamine at $37{ }^{\circ} \mathrm{C}$ in a humidified $5 \% \mathrm{CO}_{2}$ atmosphere and passaged upon reaching confluence. The use of androgen-free cell culture media resulted in no detectable levels of androgens in the collected cells. Cells were harvested using $0.05 \%$ trypsin, pelleted by centrifugation at $400 \mathrm{~g}$ for $5 \mathrm{~min}$, and washed three times by $1 \mathrm{X}$ PBS to completely remove cell culture media.

\section{LCM of biopsy samples}

Clinical tissue biopsies from recurrent PCa patients were procured from at Pathology Department of Xuanwu Hospital. Written informed consent was required for participation. The optimal cutting temperature (OCT)-embedded samples were mounted on a cryostat sample holder (Leica Microsystems, UK). The sample holder temperature was set to $-20{ }^{\circ} \mathrm{C}$ and the knife temperature to $-23{ }^{\circ} \mathrm{C}$. The tissues were sliced into $10-\mu \mathrm{m}$-thick sections, and a series of $10-\mu \mathrm{m}$ slices were mounted on POL frame slides (Leica frame slide, POL-membrane, MicroDissect GmbH, 
Germany). Before LCM, the slides were washed twice by water and then stained with Nuclear Fast Red. Excess staining reagent was washed off with water and the slides were dried by a cold air stream. The stained biopsies from PCa patients were examined and classified based on morphological features and cell type annotation by a board-certified pathologist according to the Gleason grading system. Biopsies with poor sample purity $(<95 \%)$ were disqualified, and regions of interest were marked for further evaluation.

Laser capture microdissection was performed on a Leica LMD7 Laser Microdissection System (Leica Microsystems, Wetzel, Germany) with an LMT350 ultra-scanning stage, which enables high-throughput, robust LCM dissection. The UV laser of the LMD7 system (349 nm Wavelength, $10 \mathrm{kHz}$ maximum repetition rate, and $120 \mu \mathrm{J}$ maximum pulse energy) was used for cutting tissue microdissections. The Leica Laser Microdissection software (v8.2) was used to control the microscope when taking pictures or selecting areas for laser capture microdissection and cutting.

\section{MWCO-based separation of proteins from small molecules}

Cell lysis For each LCM sample, $20 \mu \mathrm{L}$ PBS with 1\% FA was added to the collection tube, gently vortexed, and the collection tubes were wrapped with Parafilm (Sigma-Aldrich, St. Louis, MO). Samples first undergo 5 freeze-thaw cycles with liquid nitrogen, and were then sonicated with 3 sonication-cooling cycles ( $5 \mathrm{~min}$ each) in an ultrasonic water bath to lyse the cells. Cell lysates were then transferred to LoBind microcentrifuge tubes (Eppendorf, Hauppauge, NY). The lysis procedure was repeated twice with $20 \mu \mathrm{L}$ FA-acidified PBS and lysates of the same sample were combined in one tube $(\sim 60 \mu \mathrm{L}$ in total).

\section{On-membrane digestion (OMD)}

The OMD protocol was adapted from prevalently used filter-assisted sample preparation (FASP) protocols with minor optimization ${ }^{2,3}$. After re-extraction, the extracts were transferred to LoBind tubes. Disulfide bonds were reduced by adding $1 \mu \mathrm{L} 200 \mu \mathrm{M}$ DTT followed by 45 -min incubation in a covered thermomixer under $37{ }^{\circ} \mathrm{C}$ with constant agitation. Reduced proteins were diluted by $150 \mu \mathrm{L}$ Urea-Tris (UT) buffer ( $8 \mathrm{M}$ urea in $50 \mathrm{mM} \mathrm{pH} 8.5$ Tris-FA), and were transferred to 30 $\mathrm{kD}$ Amicon Ultra centrifugal filter units for 15 -min centrifugation at $10,000 \mathrm{~g}$ under room temperature (same centrifugal conditions were used in the following context unless specified otherwise). Another wash with $100 \mu \mathrm{L}$ UT buffer was repeated, and the flowthrough was then discarded after centrifugation. Subsequently, alkylation was performed by adding $50 \mu \mathrm{L} 10 \mu \mathrm{M}$ 
IAM in $50 \mathrm{mM} \mathrm{pH} 8.5$ Tris-FA followed by 45 -min incubation in a covered thermomixer under $37^{\circ} \mathrm{C}$ with constant agitation. Filters were then centrifuged, and the retentate was washed twice with $100 \mu \mathrm{L} 50 \mathrm{mM}$ pH 8.5 Tris-FA. Sample volume on filters was adjusted to $40 \mu \mathrm{L}$ using 50 $\mathrm{mM} \mathrm{pH} 8.5$ Tris-FA, and $4 \mu \mathrm{L}$ of $0.25 \mu \mathrm{g} / \mu \mathrm{L}$ trypsin prepared in $50 \mathrm{mM} \mathrm{pH} 8.5$ Tris-FA was added for overnight $(\sim 16 \mathrm{hr})$ tryptic digestion under $37{ }^{\circ} \mathrm{C}$ with constant agitation in a covered thermomixer. The digestion was terminated by adding $0.5 \mu \mathrm{L} \mathrm{FA}$. The digested sample was collected into a LoBind tube and centrifuged at $18,000 \mathrm{~g}$ under $4{ }^{\circ} \mathrm{C}$ for $30 \mathrm{~min}$. The supernatant was carefully transferred to LC vials for analysis.

\section{Global proteomic quantification}

Nano LC-Orbitrap MS. The LC-MS protocol followed an IonStar experimental procedure as described previously ${ }^{4}$. Briefly, the LC-MS system consists of a Dionex Ultimate 3000 nano LC system, a Dionex Ultimate 3000 gradient micro LC system with an WPS-3000 autosampler, and an Orbitrap Fusion mass spectrometer (ThermoFisher Scientific, San Jose, CA). A large-ID trapping column $(5 \mathrm{~mm} \times 300 \mu \mathrm{m} \mathrm{ID})$ setting was employed in front of the nano LC column (100 $\mathrm{cm} \times 75 \mu \mathrm{m}$ ID, packed with $3 \mu \mathrm{m}$ Pepmap C18) separation for high-capacity sample loading, removal of matrix components, and selective peptide delivery. Mobile phase A and B were $0.1 \%$ $\mathrm{FA}$ in $2 \% \mathrm{ACN}$ and $0.1 \% \mathrm{FA}$ in $88 \% \mathrm{ACN}$. A volume of $4 \mu \mathrm{L}$ derived peptides was loaded per injection at a flow rate of $10 \mu \mathrm{L} /$ min with $1 \%$ mobile phase $\mathrm{B}$. The nano $\mathrm{LC}$ was operated at a flow rate of $250 \mathrm{~nL} / \mathrm{min}$, with the following gradient profile: $4 \%$ to $13 \%$ B for $15 \mathrm{~min} ; 13 \%$ to $28 \% \mathrm{~B}$ for $110 \mathrm{~min}$; $28 \%$ to $44 \%$ B for $5 \mathrm{~min} ; 44 \%$ to $60 \%$ B for $5 \mathrm{~min} ; 60 \%$ to $97 \%$ B for $1 \mathrm{~min}$, isocratic at $97 \% \mathrm{~B}$ for $17 \mathrm{~min}$, and isocratic at $4 \% \mathrm{~B}$ till the end. MS was operated under data-dependent acquisition (DDA) mode, with a maximal duty cycle time of 3s. MS1 spectra were acquired in the $\mathrm{m} / \mathrm{z}$ range $400 \sim 1,500$ under $120 \mathrm{k}$ resolution with dynamic exclusion settings of 60 s exclusion durations and a mass tolerance of $\pm 10 \mathrm{ppm}$. Precursor ions were filtered by quadrupole using a 1Th window and fragmented by high energy collision dissociation (HCD) at a normalized collision energy of 35\%. MS2 spectra were acquired under 15k resolution in Orbitrap. More detailed information about the LC-Orbitrap MS system can be found in a previous report ${ }^{4}$.

Protein identification by database searching. Raw files generated by LC-MS were searched by SEQUEST-embedded Proteome Discoverer (version 1.4.1.14, Thermo Scientific) against the Homo Sapiens Swiss-Prot protein sequence database concatenated with reversed "decoy" 
sequences. The search parameters used were: i) Precursor ion mass tolerance: 20 ppm; ii) Fragment ion mass tolerance: $0.02 \mathrm{Da}$; iii) Maximal missed cleavages: 2; iv) Fixed modification: cysteine carbamidomethylation; v) Dynamic modification: methionine oxidation, peptide N-terminal acetylation. Peptide filtering, protein inference/grouping and protein false-positive discovery rate (FDR) control was performed by Scaffold (v4.3.2, Proteome Software, Inc.). Criteria for protein identification included a minimum of 2 unique peptides per protein, $<0.2 \%$ peptide FDR and $<1.0 \%$ protein FDR on the entire dataset level.

\section{Targeted androgen quantification}

Trapping-micro-LC-MS. The LC-MS method for androgen quantification was adapted and modified from a previously reported trapping-micro-LC-MS approach ${ }^{5}$. The system included an UltiMate 3000 LC system (containing SRD-3400 degasser, NCS-3500RS CAP pumps and a highflow binary gradient pump, and a WPS-3000TBRS autosampler with a $250-\mu \mathrm{L}$ loop) coupled to a TSQ Altis triple-quadrupole mass spectrometer (ThermoFisher Scientific, San Jose, CA) implemented with an H-ESI probe and a 34-G narrow-bore spray needle. Sample trapping was conducted on a C8 column $(15 \times 2.1 \mathrm{~mm}, 3.5-\mu \mathrm{m}$ particle size, $100 \AA$, Agilent, CA, USA $)$ at a flow rate of $0.4 \mathrm{~mL} / \mathrm{min}$ using the high-flow binary pump. The separation column was an Xselect CSH C18 column ( $150 \times 0.5 \mathrm{~mm}, 2.5 \mu \mathrm{m}, 130 \AA$, customized packed) at a flow rate of $25 \mu \mathrm{L} / \mathrm{min}$. A ZDV 6-port valve placed in the heated column compartment was utilized to connect the two flow systems. The mobile phase (MP) A and B were $0.1 \% \mathrm{FA}$ in $\mathrm{H}_{2} \mathrm{O}$ and $0.1 \% \mathrm{FA}$ in $85 \% \mathrm{ACN}$. For high-flow sample trapping, a volume of $8 \mu \mathrm{L}$ sample was loaded into the trapping column per injection at $25 \%$ mobile phase B for $1 \mathrm{~min}$. Then, the 16-min gradient separation on micro LC was run at a flow rate of $25 \mu \mathrm{L} / \mathrm{min}$, with the following gradient profile: $25 \%$ B for $0-1 \mathrm{~min}, 25 \%$ to $28 \%$ B for $1-6.5 \mathrm{~min}, 28 \%$ to $37.7 \%$ B for $6.5-11 \mathrm{~min}$, the trapping column was switched offline at $11 \mathrm{~min}$, and then the microflow was isocratic at $97 \% \mathrm{~B}$ for $2 \mathrm{~min}$. The detailed SRM parameters are shown in Table S1. The separation temperature was controlled at $40^{\circ} \mathrm{C}$. The spray voltage was $3.5 \mathrm{kV}$, and the capillary temperature was maintained at $325^{\circ} \mathrm{C}$. The dwell time for each transition was $50 \mathrm{~ms}$, and the resolution was set to $0.7 \mathrm{FWHM}$ for both Q1 and Q3.

Calibration and validation. Androgen-free C4-2 PCa cells were selected as the blank matrix for calibration and validation. Cells were lysed in PBS (pH 7.4) with $1 \%$ FA in a volume of $20 \mu \mathrm{L}$. Calibration samples were prepared by spiking standard compounds into C4-2 cell lysates at 5, 8, 
25, 50, 100, 200 and $500 \mathrm{fg}$ each androgen per $1 \mathrm{k}$ cells, and stable-isotope-coded internal standards were spiked at $50 \mathrm{fg} / 1 \mathrm{k}$ cells. Quality control (QC) samples were prepared by spiking androgens into C4-2 cell lysates at four levels: 5, 20, 50 and $400 \mathrm{fg} / 1 \mathrm{k}$ cells. All calibration and QC samples were processed following the experimental procedures described above. Calibration curves were established by plotting extracted ion current peak area ratio of the analyte/IS as a function of analyte concentration, using weighted least-squares linear regression analysis. The precision of the assay was calculated by repeated analysis of QC samples $(\mathrm{N}=3)$, and the coefficient of variation $(\mathrm{CV} \%)$ of the replicate measurements was calculated to determine variability. Quantitative accuracies of the QC samples at each concentration level were calculated against nominal concentrations. Calibration and validation results for each androgen quantification are shown in Table S2. 


\section{Reference}

(1) Lex, A.; Gehlenborg, N.; Strobelt, H., et al. UpSet: Visualization of Intersecting Sets. IEEE Transactions on Visualization and Computer Graphics 2014, 20, 1983-1992.

(2) Wisniewski, J. R.; Ostasiewicz, P.; Mann, M. High recovery FASP applied to the proteomic analysis of microdissected formalin fixed paraffin embedded cancer tissues retrieves known colon cancer markers. J. Proteome Res. 2011, 10, 3040-3049.

(3) Wiśniewski, J. R.; Zougman, A.; Nagaraj, N., et al. Universal sample preparation method for proteome analysis. Nat. Methods 2009, 6, 359-362.

(4) Shen, X.; Shen, S.; Li, J., et al. An IonStar Experimental Strategy for MS1 Ion Current-Based Quantification Using Ultrahigh-Field Orbitrap: Reproducible, In-Depth, and Accurate Protein Measurement in Large Cohorts. J. Proteome Res. 2017, 16, 2445-2456.

(5) Zhang, M.; An, B.; Qu, Y., et al. Sensitive, High-Throughput, and Robust Trapping-MicroLC-MS Strategy for the Quantification of Biomarkers and Antibody Biotherapeutics. Anal.

Chem. 2018, 90, 1870-1880. 